\title{
Extended Sequence and Functional Analysis of the Butirosin Biosynthetic Gene Cluster in Bacillus circulans SANK 72073
}

\author{
Fumitaka Kudo, Mario Numakura, Hideyuki Tamegai ${ }^{\dagger}$, Hideki Yamamoto, \\ Tadashi Eguchi, Katsumi Kakinuma ${ }^{\dagger \dagger}$
}

Received: February 24, 2005 / Accepted: May 10, 2005

(C) Japan Antibiotics Research Association

\begin{abstract}
Butirosin produced by Bacillus circulans is among the clinically important 2-deoxystreptamine containing aminoglycoside antibiotics and its unique structure is found in $(S)$-4-amino-2-hydroxyburyric acid substituted at C-1 of 2-deoxystreptamine. Recently, the key part of the butirosin biosynthetic gene cluster has been identified from Bacillus circulans SANK 72073, however the whole gene for the biosynthesis awaited for identification. In the present study, we undertook extended analysis of the butirosin biosynthetic gene cluster and found nine additional open reading flames (ORFs), btr $Q$, btrR1, btrR2, btrT, btrU, btrV, btrW, btrX and orf1 in the cluster. In addition, we constructed disruption mutants of btrR 1 and btrP- $V$, and found that the btr genes (ca. $24 \mathrm{~Kb})$ between $b \operatorname{tr} R 1$ and $\operatorname{btr} P-V$ are at least required for the butirosin biosynthesis.
\end{abstract}

Keywords 2-deoxystreptamine, butirosin, biosynthesis, gene cluster, gene disruption, Bacillus circulans

T. Eguchi (Corresponding author): Department of Chemistry and Materials Science, Tokyo Institute of Technology, 2-12-1, O-okayama, Meguro-ku, Tokyo 152-8551, Japan, E-mail: eguchi@cms.titech.ac.jp

F. Kudo, M. Numakura, H. Tamegai, H. Yamamoto, K. Kakinuma: Department of Chemistry, Tokyo Institute of Technology, 2-12-1, O-okayama, Meguro-ku, Tokyo 152-8551, Japan

${ }^{\dagger}$ Present address: Department of Chemistry, College of Humanities and Sciences, Nihon University, 3-25-40 Sakurajosui, Setagaya-ku, Tokyo 156-8550, Japan

${ }^{\dagger \dagger}$ Deceased April 1, 2005

\section{Introduction}

Butirosin produced by Bacillus circulans is among the clinically important 2-deoxystreptamine (DOS)-containing aminoglycoside antibitoics [1]. However, the rapid emergence of resistant bacteria to this class of antibiotic has urged us to develop structurally novel and more potent antibiotics [2 4]. In this respect, butirosin is unique in its activity against resistant bacteria due to the substitution with $(S)$-4-amino-2-hydroxybutyryl moiety at C-1 of DOS. This feature allowed to develop amikacin as a commercial semisynthetic antibiotic for the treatment of resistant bacteria [5]. Biosynthetic approach appears to be an alternative to this end $[6,7]$.

Concerning to the biosynthesis of butirosin, isotopefeeding experiments and cosynthesis methods using blocked mutants allowed to elucidate the gross biosynthetic pathway [8,9]. More recently, a significant part of the butirosin biosynthetic gene cluster has been identified from Bacillus circulans SANK 72073 through the discovery of the protein (BtrC) and of the gene $(b t r C)$ for 2-deoxyscyllo-inosose synthase, which catalyzes the key carbocyclization of glucose-6-phosphate in the pathway for DOS (Scheme 1) [10 12]. More recently last year, based on the information of $b \operatorname{tr} C$ and resistance genes, the biosynthetic gene clusters for three other DOS containing aminoglycosides have been identified [13 18]. Functional analysis of $b \operatorname{tr} C, b \operatorname{tr} S$ and $b \operatorname{tr} D$ has successfully shown that this btr gene cluster is involved in the butirosin biosynthesis $[10,12,19 \sim 21] . \quad B t r S$ is an unique aminotransferase involved dually in the pathway for DOS [20]. BtrD is a novel enzyme involved in the biosynthesis of dTDP-glucosamine from dTTP and glucosamine-1- 

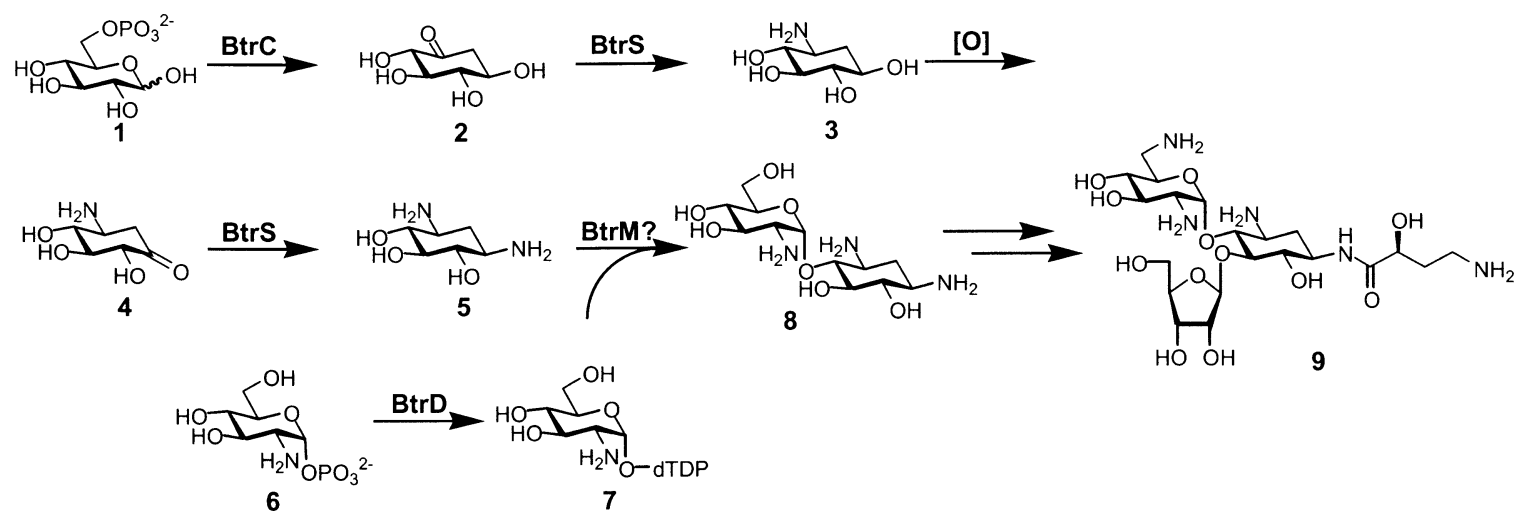

Scheme 1 Biosynthesis of butirosin.

1, D-glucose-6-phosphate; 2, 2-deoxy-scyllo-inosose; 3, 2-deoxy-scyllo-inosamine; 4, aminodideoxy-scyllo-inosose; 5, 2deoxystreptamine; 6, D-glucosamine-1-phosphate; 7, dTDP-glucosamine; 8, paromamine; 9, butirosin B. BtrC, 2-deoxy-scyllo-inosose synthase; BtrS, L-glutamine:2-deoxy-scyllo-inosose aminotransferase; BtrD, dTDP-glucosamine synthase; BtrM, putative glycosyltransferase

phosphate prior to the glycosylation in the paromamine synthesis [21]. Although seventeen open reading frames (ORFs) have been reported so far [11, 12], all the required genes for the butirosin biosynthesis have not yet been determined. Thus, in order to address this important issue, we have pursued extended analysis of the butirosin biosynthetic gene cluster toward upstream and downstream, and nine additional open reading flames (ORFs), btrQ, btrR1, btrR2, btrT, btrU, btrV, btrW, btrX and orf1 have been supplemented to the cluster. The DNA sequence between $b \operatorname{tr} R 1$ to $\operatorname{btr} T$ was independently deposited by Huang et al. in different codes names derived from a different type strain of B. circulans NRRL B3312 (AJ494863). In the present study, we have further carried out functional analysis of appropriate genes in the btr gene cluster by gene disruptions and found that the btr genes from $b t r R 1$ through $b t r P-V$ are at least required for the butirosin biosynthesis.

\section{Materials and Methods}

\section{General}

Bacillus circulans SANK 72073 was used as the source strain for butirosin biosynthetic genes and was also used for the construction of gene disruption mutants. Escherichia coli JM 109 and DH5 $\alpha$ were used as the host strain for btr gene cloning. Bacillus subtilis PCI 219 was used as an antibiotic test strain. The plasmids for gene disruption were constructed with an E. coli-Bacillus shuttle vector pHB201 (Tanaka et al., unpublished). Also routinely used was pUC 119 vector for sub-cloning and sequencing. Another vector pT7-blue T (Novagen, USA) was routinely used for sub- cloning after PCR. DNA sequence analysis was carried out with a LONG READER 4200 (Li-Cor) according to the manufacturer's protocol. Other reagents were the highest grade commercially available.

\section{Gene Walking}

Standard in vitro techniques were used for DNA manipulation [22]. Gene walking toward the upstream of the known region was carried out by inverse PCR. Based on the previously determined sequence [12], two oligonucleotides btrS1: 5'-TTATCGCCCATCTGTTCGACTGAAA-3' and btrS2: 5'-CGCGAGGCAATGGAAGAACAAATTA-3' were designed in inverse direction as primers. EcoRI-digested chromosomal DNA of B. circulans was, after the phenol-chloroform extraction, self-ligated. The resulting circular DNAs were used as PCR template. The PCR conditions were; 1 cycle at $95^{\circ} \mathrm{C}$ for 10 minutes, followed by 30 cycles of $95^{\circ} \mathrm{C}$ for 1 minute, $55^{\circ} \mathrm{C}$ for 1 minute, $72^{\circ} \mathrm{C}$ for 5 minutes, and then $72^{\circ} \mathrm{C}$ for 7 minutes, using AmpliTaq Gold DNA polymerase (Applied Biosystems, USA). The PCR product was subcloned into pT7-blue T vector, and the resulting plasmid pDS17 was sequenced. Subsequently, pDS18, 19, 20, and 21 were obtained similarly, using HincII-, Eco RI-, HincII-, and StuIdigested circular DNA fragments as template, respectively and primers pDS18-1: 5'-GCGTACCGTAATGTTTCGGTTCC-3' and pDS18-2: 5'-CGGATTGTGCGTAGAAGCAGAAG-3' for pDS18, pDS19-1: 5'-GCAAAGCCTGCTCCCGCCCGATA-3' and pDS19-2: 5'-CGAGGCGGGCGGCCTGATCGGGT-3' for pDS19, pDS20-1: 5' AACGGACGACTCCACCACCTGAG-3' and pDS20-2: 5'-CGTTAGTCGTCGTGGCTCATCCG-3' for pDS20, pDS21-1 5'-CGACATAGACCTGAACAGCCGCC-3' and 
pDS20-2 for pDS21.

Gene walking to the downstream region was carried out with Southern hybridization and inverse PCR. SacI-SalI fragment was prepared from pDS14 [11], and the fragment was digoxigenin (DIG)-labelled with DIG DNA labeling kit (Roche Applied Science, Switzerland) for a probe. DraIdigested chromosomal DNA was subcloned into SmaI site of pUC 119 to derive a library. Hybridization was carried out using the probe in a standard manner, and a positive clone containing pDS15 was obtained and then sequenced. In order to analyze the downstream of pDS15, pDS22 were also obtained by inverse PCR as described above, using $P v u \mathrm{I}$-digested and self-ligated chromosomal DNA as a template. The primers, pDS22-1 5'-CGGTTTGGGAAGACGGATAGCGG-3' and pDS22-2 5'-CCGGCAGCGCACCGTTCGGCAGG-3' were designed from the information of $\mathrm{pDS} 15$ sequence.

The sequences determined in the present study have been deposited in DDBJ/EMBL/GenBank nucleotide sequence databases with the accession numbers of AB097196.

\section{Disruption of Key btr Genes Disruption of btrR1}

DNA fragments containing a part of btrRl gene, from 107 to 930 ( $823 \mathrm{bp}$ ) and from 1485 to 2327 ( $842 \mathrm{bp}$ ), were amplified by PCR with two sets of primers btrR1-d1: $5^{\prime}$ TGGATCCCCGGCAGGCGGACCCTCGGTTGG-3' and btrR1-d2: 5'-CGCCGGCCGTTTGCGCTGATTCCGGATCGC-3', btrR1-d3: 5'-AGCCGGCGACTCAGGTGGTGGAGTCGTCCG-3' and btrR1-d4: 5'-TGGATCCCTGTGCTCATGTCCGATTCCTCC-3' containing BamHI and NaeI sites, and subsequently sub-cloned into pT7-blue $\mathrm{T}$ vector. After the confirmation of the sequences, the two DNA fragments were so ligated as to construct an in-frame disrupted btrR1 gene fragment, which was then cloned into a BamHI site of pUC119. After recovery of amplified plasmid, the BamHI fragment containing in-frame disrupted btrRl was excised and then inserted into the corresponding restriction site of pHB201. The resulting plasmid pHBbtrR1dlt was introduced into wild type cells of B. circulans SANK 72073 by electroporation (Easyjet Optima, EquiBio, UK), and btrRl gene disruptant was constructed by homologous recombination according to the previously described method [11]. Disruption of the btrR1 gene was confirmed by PCR with primers btrR1-f: 5'-GCGATCCGGAATCAGCGCAAACG-3' and btrR1d5: 5'-GTCCATGTAATCGCCCGACCGGA-3' for the amplification of the DNA containing btrR1. PCR conditions were $95^{\circ} \mathrm{C}, 5$ minutes for denature, 30 cycles of $95^{\circ} \mathrm{C}, 1$ minute, $40^{\circ} \mathrm{C}, 1$ minute, $72^{\circ} \mathrm{C}, 3$ minutes for extension of DNA.
The btrRl disruptant was cultured in a glycerolsupplemented nutrient broth. An aliquot of the culture was collected every day for 4 days, and antibiotic activity of each supernatant was tested by a paper disk diffusion method against B. subtilis PCI 219.

To complement the btrRl mutation, a whole btrRl gene was first amplified by PCR with primers R1-sf: 5' CCTGGATCCGTAACCTAGGGC-3' and R1-sr: 5'AATACCTTCTCCGGATCCGCG-3' and a template $B$. circulans chromosomal DNA. PCR conditions were $95^{\circ} \mathrm{C}$, 1 minute for denature, 30 cycles of $95^{\circ} \mathrm{C}, 30$ seconds, $60^{\circ} \mathrm{C}, 45$ seconds, $72^{\circ} \mathrm{C}, 30$ seconds for extension of DNA. The amplified DNA fragment was sub-cloned into pT7-blue $\mathrm{T}$ vector. After confirmation of the sequence, the BamHI fragment was inserted into the corresponding restriction site of pHB201 to yield an expression plasmid pHBRs, which was introduced by electroporation into the abovementioned btrRl disruptant for complementation. The complemented strain was cultured in the above-mentioned glycerol-supplemented nutrient broth containing $1 \mu \mathrm{g} / \mathrm{ml}$ of erythromycin. An aliquot of the culture was collected everyday and its supernatant was tested on antibiotic activity by a paper disk diffusion method against $E$. coli JM109. The supernatant of a 5-days-culture was loaded onto a column of Amberite IRC-50 $\left[\mathrm{NH}_{4}^{+}\right]$resin, which was subsequently washed with water and $0.1 \mathrm{M} \mathrm{NH}_{4} \mathrm{OH}$, and then butirosin-containing fractions were eluted with $1 \mathrm{M}$ $\mathrm{NH}_{4} \mathrm{OH}$. After concentration of the fractions, the residue was separated by TLC (Merck silica gel $60 \mathrm{~F}_{254}, 0.25 \mathrm{~mm}$ thick, $20 \mathrm{~cm} \times 20 \mathrm{~cm}$ ) with a solvent $\mathrm{CHCl}_{3}: \mathrm{MeOH}$ : $\mathrm{NH}_{4} \mathrm{OH}: \mathrm{EtOH}=4: 6: 7: 1$. The antibiotics separated by TLC were directly transferred onto the agar plate containing spore of B. subtilis PCI 219. B. subtilis was cultured at $37^{\circ} \mathrm{C}$ overnight and the detected antibiotic was compared with standard butirosin.

\section{Disruption Around btrP-V}

The $b \operatorname{tr} P-V$ disruptant was constructed by the same manner as above mentioned with the primers btrP-d1: $5^{\prime}-$ TGGATCCTGCTTACGGCATAGTCGGGTCCC-3', btrPd2: 5'-CAGATCTATATTGCAGCGGGCCtGCGCATG-3', btrP-d3: 5'-GAGATCTGTGGTCTACGCGATTTCCGGCGT-3' and btrP-d4: 5'-AGAATTCCGCTTTGGATGGCGTTACCGGCC-3'. The mutant was screened by PCR with btrP-d5: 5'-CATGCGCAGGCCCGCTGCAATAT-3' and btrP-d6: 5'-GTTCCCTACTCCGTCCGGCCACC-3'.

\section{RT-PCR}

B. circulans RNA was prepared with RNAprotect ${ }^{\mathrm{TM}}$ Bacteria Reagent (Qiagen) and RNeasy Mini Kit (Qiagen). RT-PCR was performed with Qiagen OneStep RT-PCR Kit 
(Qiagen) using btrR1-f: 5'-GCGATCCGGAATCAGCGCAAACG-3' and btrR1-r: 5'-TTGCCATCCATTCCTGCTGGGCC-3' for btrR1, btrU-f: 5'-GCAGCAGTCCCGTGTCAACAAAC-3' and btrU-r: 5'-GCAATCCGGGGATCAAGCTCGGG-3' for btrU, btrR1U-f: 5'GGCCCAGCAGGAATGGATGGCAA-3' and btrR1U-r: 5'-GTTTGTTGACACGGGACTGCTGC-3' for the region between btrRl and btrU, OP-f: 5'-GGCGGAGCAAGCATGGAAGATCG-3' and OP-r: 5'-CCCGGAGCTTCACGGCCTGGCCG-3' for the region between $b$ trO and btrP, PV-f: 5'-CGGCCAGGCCGTGAAGCTCCGGG-3' and PV-r: 5'-ACGCCGGAAATCGCGTAGACCAC-3' for the region between $b \operatorname{tr} P$ and $b \operatorname{trV}$, VQ-f: 5'-GTGGTCTACGCGATTTCCGGCGT-3' and VQ-r: 5'-CCGCCGCCAACCGCGCTGGCAGA-3' for the region between $b \operatorname{tr} V$ and $b \operatorname{tr} Q$, according to the manufacturer's protocol. The PCR conditions were; 1 cycle at $50^{\circ} \mathrm{C}$ for 30 minutes and $94^{\circ} \mathrm{C}$ for 5 minutes, followed by 40 cycles of $94^{\circ} \mathrm{C}$ for 1 minute, $60^{\circ} \mathrm{C}$ for 1 minute, $72^{\circ} \mathrm{C}$ for 1 minute, and then $72^{\circ} \mathrm{C}$ for 10 minutes.

\section{Western Blotting}

The lysis of B. circulans was carried out with final $2 \%$ of SDS and $2 \%$ of mercaptoethanol. After standard SDSPAGE, the proteins were transferred onto PVDF membrane by electro-blotting (horizeblot, ATTO). The membrane was soaked in a solution containing BtrC polyclonal antibody with immunblotting kit (Bio-Rad). The expression of BtrC in $B$. circulans was visualized with horseradish peroxidase detection kit (Bio-Rad).

\section{Results and Discussion}

\section{Extended Analysis of $\boldsymbol{b t r}$ Gene Cluster}

Gene walking toward the upstream of the so far established btr gene cluster was carried out by inverse PCR in order to figure out the starting region, since all the known ORFs are aligned in normal direction. Four additional hypothetical ORFs, btrR1, btrU, btrR2, btrT and a partial ORF orf1 were found in the upstream of the $b t r S$ gene (Table 1 and Figure 1). The orfl gene at the far end showed homology to a gene encoding a hypothetical protein of $B$. halodurans and was identified in the opposite direction to all other $b t r$ genes. Therefore, orfl appears to be in a different operon. It should be pointed out then that a consensus promoter sequence was found in the upstream of btrRl (Figure 1) [23]. Thus, an operon starting the btrRl gene was envisioned to be the butirosin biosynthetic gene cluster. In fact, the deduced amino acid sequence of btrRl showed significant homology to TetR transcriptional regulator family [24]. In addition, the deduced amino acid sequence of $b t r R 2$ showed high homology to the hypothetical protein of Burkholderia cepacia and suppressors of fused $(\mathrm{Su}(\mathrm{fu}))$ in human, mouse, and Drosophila, which function as a negative regulator of the Hedgehog segment polarity pathway by physically interacting with several proteins in the signaling pathway [25 29]. 3D-PSSM search [30, 31] for a model structure of BtrR2 also showed a high similarity to the $\mathrm{Su}(\mathrm{fu})$ of human with low $\mathrm{E}$ value. Thus, BtrR2 may interact with butirosin biosynthetic proteins and suppress the activity in tropophase. Consequently, it may be

Table 1. Summary of the newly identified btr genes and comparison with database

\begin{tabular}{|c|c|c|c|}
\hline ORF & Size bp (aa) & Homological proteins, size of protein (aa), source, tag number & Homology (aa/aa, \%) \\
\hline btr $P$ & $642(214)$ & fructose-2,6-bisphosphatase, 199 aa, Lactobacillus gasseri, ZP 00046611 & $48 / 165,29 \%$ \\
\hline \multirow[t]{3}{*}{$b \operatorname{tr} Q$} & $1371(457)$ & hypothetical protein, 508 aa, S. tenebrarius, CAE22474 & $195 / 451,43 \%$ \\
\hline & & $\begin{array}{l}\text { putative sugar-alcohol dehydrogenase, } 507 \text { aa, Micromonospora echinospora, } \\
\text { AAR98543 }\end{array}$ & $194 / 457,42 \%$ \\
\hline & & oxidoredcutase, 508 aa, S. kanamyceticus, BAD20757 & $191 / 451,42 \%$ \\
\hline$b t r R 1$ & $654(218)$ & $\begin{array}{l}\text { putative tetR-family transcriptional regulatory protein, } 233 \text { aa, S. coelicolor A3(2), } \\
\text { NP } 625398\end{array}$ & $41 / 99, \quad 41 \%$ \\
\hline \multirow[t]{2}{*}{$b t r R 2$} & $1143(381)$ & hypothetical protein, 362 aa, Burkholderia cepacia R18194, ZP 00212925 & $166 / 358,46 \%$ \\
\hline & & Suppressor of Fused, 433 aa, Homo sapiens, AAF23890 & $103 / 301,34 \%$ \\
\hline$b t r T$ & $234(78)$ & no typical homology & \\
\hline$b \operatorname{trU}$ & $576(192)$ & putative NAD(P)H oxidoreductase, 197 aa, Bacillus subtilis, NP 388411 & $91 / 184,49 \%$ \\
\hline$b t r V$ & $249(83)$ & $\begin{array}{l}\text { hypothetical protein, } 117 \text { aa, Photorhabdus luminescens subsp. Laumondii TTO1, } \\
\text { NP } 931337\end{array}$ & $26 / 77, \quad 33 \%$ \\
\hline$b t r W$ & $1758(586)$ & putative ABC transporter, 585 aa, B. subtilis, NP 388852 & $309 / 576,53 \%$ \\
\hline$b \operatorname{tr} x$ & $>1375(>459)$ & putative ABC transporter, 666 aa, B.cereus, ZP 00235844 & $227 / 458,49 \%$ \\
\hline orf1 & $>514(>172)$ & hypothetical protein BH3120, 163 aa, B. halodurans C-125, BAB06839 & $41 / 150,27 \%$ \\
\hline
\end{tabular}


a)

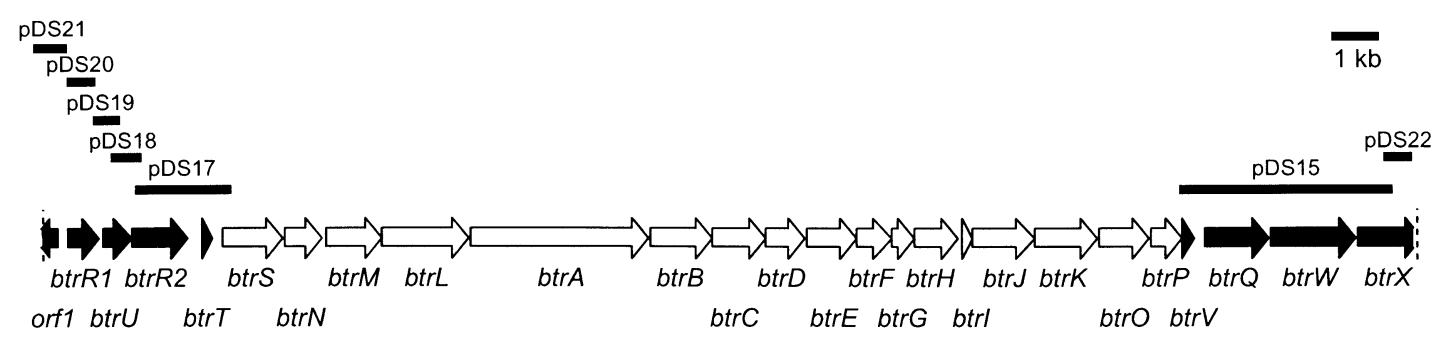

b)

AACCTAGgGCCTGgCGgTTGACAACACCGTTTTATTTTTTCTATAATGGCATGG

ATTTTAAAGACGGAACTAAAGGTGAAGCGGCATGTCTCCACGTACTAAGGAACA

Fig. 1 a) Genetic map of the btr gene cluster. Black arrow indicates the ORFs found in the present study. b) Upstream of btrR1. Boxes indicate the consensus promoter sequences.

proposed that the combination of BtrR1 and BtrR2 may regulate the butirosin biosynthesis. The deduced btrU gene product showed a high homology to $\mathrm{NAD}(\mathrm{P}) \mathrm{H}$ oxidoreductase of various microorganisms. The $b \operatorname{tr} T$ gene was found coding a short protein with no typical homology.

Gene walking to the downstream was carried out with Southern hybridization and inverse PCR. Three additional hypothetical ORFs, $b \operatorname{tr} V, b \operatorname{tr} Q, b t r W$ and a partial ORF $b \operatorname{tr} X$ were found in this order under the $b \operatorname{tr} P$ gene. It should be pointed out that the previously reported $b \operatorname{tr} P$ gene [11] has to be revised, since an independent ORF btrV was newly found between $\operatorname{btr} P$ and $b \operatorname{tr} Q$ as shown in Figure 1. Interestingly, the deduced BtrV showed quite high homology to hypothetical protein of Photorhabdus luminescens subsp. Laumondii TTO1 (NC_005126), apparently residing adjacent to a $b \operatorname{tr} A$ homologue in the genome of Photorhabdus luminescens. Therefore, BtrA and BtrV may have certain role in combination for the butirosin biosynthesis. The $N$-terminus amino acid of the deduced BtrP showed a similarity to fructose-2,6-bisphosphatases/ phosphoglycerate mutases of various microorganisms. Interestingly, the deduced amino acid of $b \operatorname{tr} Q$ gene has high homology to the genes encoding putative dehydrogenase in the DOS-containing aminoglycoside biosynthetic gene cluster, including a tobramycin producer $S$. tenebrarius (AJ579650) [13], a gentamicin producer Micromonospora echinospora (AY524043) [15] and a kanamycin producer Streptomyces kanamyceticus (AB164142) [17]. BtrQ appears to have close relation to the DOS containing aminoglycoside biosynthesis. The other two genes btr $W$ and $\operatorname{btr} X$ showed significant homology with the genes encoding putative ABC transporters of many species. Aubert-Pivert and Davies previously showed the existence of an bacterial surface protein gene butB and a hypothetical protein gene orfB adjacent to an aminoglycoside phosphotransferase gene butA in B. circulans NRRL B3312 [32]. Based on the finding that the insertional mutation of butB caused the defection in antibiotic production, they suggested that ButB plays a role in butirosin-export to the outside of the cells. Therefore, the putative transporters btr $W$ and $b t r X$ in the btr gene cluster could be related to the exporting system either in combination with the But protein or directly related to the resistance because these genes are obviously in the biosynthetic gene cluster.

\section{Disruption of the btr Genes}

In order to confirm the relation of the newly identified genes to the butirosin biosynthesis, the btrRl gene was first disrupted. The deduced amino acid sequence of btrRl showed the significant homology to TetR transcriptional regulator family as mentioned above. Since TetR binds tet $O$, which in turn represses the transcription of tet $A$ tetracycline resistance gene, we anticipated that BtrR1 would bind the DNA sequence responsible to the transcription of butirosin biosynthetic genes and regulate the butirosin production. In addition, 3D-PSSM search for the model structure of BtrR1 also showed a high similarity toward TetR transcriptional regulators with low E value. In this hypothetical structure, the $N$-terminus of BtrR1 clearly appeared as a helix-turn-helix motif well conserved as a DNA binding site in the TetR family. Subsequently, we pursued disruption studies of the btrRl gene. The btrRl disrupted strain did not show any antibiotic production, while the btrRl gene complementation recovered the butirosin production. Thus, BtrR1 was found to work as an activator for butirosin biosynthesis in B. circulans. Further, 
in the btrRl disruptant, the transcription of a region $\operatorname{btr} V$ to $b t r Q$ was confirmed by RT-PCR. Also, the expression of BtrC was confirmed by Western blotting. Thus, BtrR1 appears to regulate the butirosin biosynthesis, however, the actual target is not clear at the moment. It should be interesting to identify the detailed molecular mechanism of BtrR1 regulation for the butirosin biosynthesis.

In-frame disruption of $b t r Q$ locating for a downstream of the cluster has been attempted without success. However, the contiguous two genes $b \operatorname{tr} P-V$ just nextmost to $b \operatorname{tr} Q$ was disrupted instead. As a result, the mutant did not show any antibiotic production either. Therefore, $b \operatorname{tr} P$ and/or $b t r V$ must be required for the butirosin biosynthesis. In addition to the obvious homology of BtrQ to the genes found in the other DOS-containing aminoglycoside producers, the btr genes from btrRl to btr $Q$ would be necessary for the butirosin biosynthesis.

\section{Transcriptional Analysis by RT-PCR}

We then investigated the range of transcription of $b t r$ genes around the upstream and downstream regions. Since there is a promoter like sequence between btrRl and btrU, RTPCR was carried out with the mRNA extracted from $B$. circulans at the early stage of growth. As a result, the RNA corresponding to btrR1, btr $U$ and the sequence between $b t r R 1$ and $b t r U$ have been already transcribed after 6 hours of fermentation. The RNAs between $b \operatorname{tr} O$ and $b \operatorname{tr} P, b \operatorname{tr} P$ and $b \operatorname{tr} V, b \operatorname{tr} V$ and $\operatorname{btr} Q$ were similarly analyzed by RTPCR. As a result, all the sequences were amplified, so that this region should be within a $b t r$ operon.

In summary, eight additional btr genes have been identified in the butirosin biosynthetic cluster in $B$. circulans and the genes between btrR1-btrP-V are at least required for the butirosin biosynthesis, although we were not able to conclude the range of btr operons. In addition, BtrR1 was clearly found as one of regulatory protein in the biosynthesis of butirosin based on the disruption study. Further analysis of the full btr cluster and the regulation mechanism seem necessary to elucidate the whole scenario of the butirosin biosynthesis.

Acknowledgement This work was supported in part by a Grantin-Aid for Scientific Research and a COE21 program from a Ministry of Education, Culture, Sports, Science and Technology of Japan.

\section{References}

1. Dion HW, Woo PW, Willmer NE, Kern DL, Onaga J, Fusari SA. Butirosin, a new aminoglycosidic antibiotic complex: isolation and characterization. Antimicrob Agents Chemother 2: 84-88 (1972)

2. Magnet S, Blanchard JS. Molecular insights into aminoglycoside action and resistance. Chem Rev 105: 477497 (2005)

3. Vakulenko SB, Mobashery S. Versatility of aminoglycosides and prospects for their future. Clin Microbiol Rev 16: 430 450 (2003)

4. Llano-Sotelo B, Azucena EF, Jr, Kotra LP, Mobashery S, Chow CS. Aminoglycosides modified by resistance enzymes display diminished binding to the bacterial ribosomal aminoacyl-tRNA site. Chem Biol 9: 455-463 (2002)

5. Naito $T$, Nakagawa $S$, Narita $Y$, Toda $S$, Abe $Y$. Aminoglycoside antibiotics. IX. 1-N-acyl derivatives of kanamycin A (amikacin analogs). J Antibiot 27: 851-858 (1974)

6. Rinehart KL, Jr. Biosynthesis and mutasynthesis of aminocyclitol antibiotics. J Antibiot 32 Suppl.: S32-46 (1979)

7. Daum SJ, Lemke JR. Mutational biosynthesis of new antibiotics. Annu Rev Microbiol 33: 241-265 (1979)

8. Takeda K, Aihara K, Furumai T, Ito Y. Biosynthesis of butirosins. I. Biosynthetic pathways of butirosins and related antibiotics. J Antibiot 32: 18-28 (1979)

9. Furumai T, Takeda K, Kinumaki A, Ito Y, Okuda T. Biosynthesis of butirosins. II. Biosynthetic pathway of butirosins elucidated from cosynthesis and feeding experiments. J Antibiot 32: 891-899 (1979)

10. Kudo F, Tamegai H, Fujiwara T, Tagami U, Hirayama K, Kakinuma K. Molecular cloning of the gene for the key carbocycle-forming enzyme in the biosynthesis of 2deoxystreptamine-containing aminocyclitol antibiotics and its comparison with dehydroquinate synthase. J Antibiot 52: 559-571 (1999)

11. Ota Y, Tamegai H, Kudo F, Kuriki H, Koike-Takeshita A, Eguchi T, Kakinuma K. Butirosin-biosynthetic gene cluster from Bacillus circulans. J Antibiot 53: 1158-1167 (2000)

12. Tamegai H, Nango E, Kuwahara M, Yamamoto H, Ota Y, Kuriki H, Eguchi T, Kakinuma K. Identification of L-glutamine:2-deoxy-scyllo-inosose aminotransferase required for the biosynthesis of butirosin in Bacillus circulans. J Antibiot 55: 707-714 (2002)

13. Kharel MK, Subba B, Lee HC, Liou K, Woo JS, Sohng JK. An approach for cloning biosynthetic genes of 2deoxystreptamine-containing aminocyclitol antibiotics: isolation of a biosynthetic gene cluster of tobramycin from Streptomyces tenebrarius. Biotechnol Lett 25: 2041-2047 (2003)

14. Kharel MK, Basnet DB, Lee HC, Liou K, Woo JS, Kim BG, Sohng JK. Isolation and characterization of the tobramycin biosynthetic gene cluster from Streptomyces tenebrarius. FEMS Microbiol Lett 230: 185-190 (2004)

15. Unwin J, Standage S, Alexander D, Hosted T, Jr., Horan AC, Wellington EM. Gene cluster in Micromonospora echinospora ATCC15835 for the biosynthesis of the 
gentamicin C complex. J Antibiot 57: 436-445 (2004)

16. Kharel MK, Basnet DB, Lee HC, Liou K, Moon YH, Kim JJ, Woo JS, Sohng JK. Molecular cloning and characterization of a 2-deoxystreptamine biosynthetic gene cluster in gentamicin-producing Micromonospora echinospora ATCC15835. Mol Cells 18: 71-78 (2004)

17. Yanai K, Murakami T. The kanamycin biosynthetic gene cluster from Streptomyces kanamyceticus. J Antibiot 57: 351-354 (2004)

18. Kharel MK, Subba B, Basnet DB, Woo JS, Lee HC, Liou K, Sohng JK. A gene cluster for biosynthesis of kanamycin from Streptomyces kanamyceticus: comparison with gentamicin biosynthetic gene cluster. Arch Biochem Biophys 429: 204-214 (2004)

19. Huang F, Li Y, Yu J, Spencer JB. Biosynthesis of aminoglycoside antibiotics: cloning, expression and characterisation of an aminotransferase involved in the pathway to 2-deoxystreptamine. Chem Commun 2860-2861 (2002)

20. Yokoyama K, Kudo F, Kuwahara M, Inomata T, Tamegai H, Eguchi T, Kakinuma K. Stereochemical recognition of doubly functional aminotransferase in 2-deoxystreptamine biosynthesis. J Am Chem Soc 127: 5869-5874 (2005)

21. Kudo F, Kawabe K, Kuriki H, Eguchi T, Kakinuma K. A new family of glucose-1-phosphate/glucosamine-1phosphate nucleotidylyltransferase in the biosynthetic pathways for antibiotics. J Am Chem Soc 127: 1711-1718 (2005)

22. Sambrook J, Fritsch EF, Maniatis T. Molecular cloning: A laboratory manual, 2nd edn. Cold Spring Harbor Laboratory, New York, (1989)

23. Haldenwang WG. The sigma factors of Bacillus subtilis. Microbiol Rev 59: 1-30 (1995)

24. Orth P, Schnappinger D, Hillen W, Saenger W, Hinrichs W.
Structural basis of gene regulation by the tetracycline inducible Tet repressor-operator system. Nat Struct Biol 7: 215-219 (2000)

25. Cohen MM, Jr. The hedgehog signaling network. Am J Med Genet A 123: 5-28 (2003)

26. Nybakken K, Perrimon N. Hedgehog signal transduction: recent findings. Curr Opin Genet Dev 12: 503-511 (2002)

27. Stone DM, Murone M, Luoh S, Ye W, Armanini MP, Gurney A, Phillips H, Brush J, Goddard A, De Sauvage FJ, Rosenthal A. Characterization of the human suppressor of fused, a negative regulator of the zinc-finger transcription factor Gli. J Cell Sci 112: 4437-4448 (1999)

28. Kogerman P, Grimm T, Kogerman L, Krause D, Unden AB, Sandstedt B, Toftgard R, Zaphiropoulos PG. Mammalian suppressor-of-fused modulates nuclear-cytoplasmic shuttling of Gli-1. Nat Cell Biol 1: 312-319 (1999)

29. Ding Q, Fukami S, Meng X, Nishizaki Y, Zhang X, Sasaki H, Dlugosz A, Nakafuku M, Hui C. Mouse suppressor of fused is a negative regulator of sonic hedgehog signaling and alters the subcellular distribution of Glil. Curr Biol 9: 11191122 (1999)

30. Fischer D, Barret C, Bryson K, Elofsson A, Godzik A, Jones D, Karplus KJ, Kelley LA, MacCallum RM, Pawowski K, Rost B, Rychlewski L, Sternberg M. CAFASP-1: critical assessment of fully automated structure prediction methods. Proteins Suppl 3: 209-217 (1999)

31. Kelley LA, MacCallum RM, Sternberg MJ. Enhanced genome annotation using structural profiles in the program 3D-PSSM. J Mol Biol 299: 499-520 (2000)

32. Aubert-Pivert E, Davies J. Biosynthesis of butirosin in Bacillus circulans NRRL B3312: identification by sequence analysis and insertional mutagenesis of the butB gene involved in antibiotic production. Gene 147: 1-11 (1994) 